\title{
Safe and Secure
}

\section{Dear Reader,}

Today's highly complex vehicle software is necessary to enable the step to be taken towards piloted driving. At the same time, however, it represents a risk for vehicle occupants and other road users that must not be underestimated. Software and hardware must not only be exceptionally reliable and updatable, they must also be protected against attack by hackers.

Up to a hundred computers are in operation in modern cars. Hundreds of millions of code characters are sent back and forth through the vehicle's network. And the quantity of software will increase even further with the introduction of partially or fully automated driving. Inevitably, this will also lead to a rise in the probability of error. This will be particularly critical wherever the effects of car-to-X communication and piloted driving come into play. Attacks by hackers such as those against the Uconnect infotainment system in the Jeep Cherokee and the security vulnerability in the Onstar RemoteLink iOS app from GM are clear illustrations of the acute need to take action. The magazine "wired" reported that hackers have succeeded in using a smartphone app and a laptop to take control of a Jeep Cherokee while it was on the move, while in another incident they managed to unlock a GM vehicle and start the engine.

Industry representatives and Germany's Federal Highway Research Institute (BASt) are aware of the problem and emphasise that security and safety experts need to jointly determine and assess risks even before the development of hardware and software begins. However, there is still a considerable need to catch up when it comes to the standardi- sation and testing of IT security relating to car-to-X communication - above all because the in-vehicle systems still lack the necessary upgrade and migration capability, as insiders already analysed as part of the ITS corridor test between Rotterdam and Vienna.

Assistance systems are in a way imperfect. And to a certain extent they will always remain so. What is important is not to raise public expectations too much. Automated vehicles do not make driving safer in itself. That is the job of assistance systems. They can only ease the burden on drivers - and not make their driving ability worse than it is today.

Best regards,

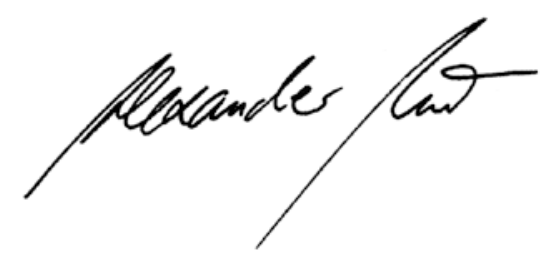

Dr. Alexander Heintzel

Editor in Chief

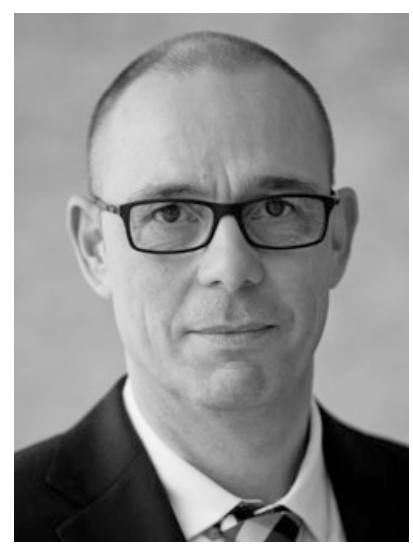

